\title{
MicroRNA-34a inhibits cell proliferation and induces cell apoptosis of glioma cells via targeting of Bcl-2
}

\author{
JUNWEI DUAN $^{1 *}$, KEJUN ZHOU $^{2 *}$, XIAOPING TANG $^{1}$, JIE DUAN $^{1}$ and LONG ZHAO ${ }^{1}$ \\ Departments of ${ }^{1}$ Neurosurgery and ${ }^{2}$ Pediatric Surgery, The Affiliated Hospital of North Sichuan Medical College, \\ Nanchong, Sichuan 637000, P.R. China
}

Received May 15, 2015; Accepted March 30, 2016

DOI: $10.3892 / \mathrm{mmr} .2016 .5255$

\begin{abstract}
Glioblastoma is a highly malignant brain tumor, characterized by the poor prognosis and high recurrence rates. Despite therapeutic strategies including surgery, radiotherapy and chemotherapy, the median survival of patients is only 14.6 months. MicroRNAs (miRNAs) have been considered as a novel type of gene regulator. Previous studies have demonstrated that the expression of miRNA-34a (miR-34a) is significantly associated with the grade and prognosis of glioma. However, the exact function of miR-34a on glioma progression and underlying mechanisms remain to be elucidated. The present study investigated the function of miR-34a in U87 human glioma cells by exogenously transfecting cells with an miR-34a mimic. Overexpression of miR-34a inhibited proliferation, and induced apoptosis of U87 cells. The current study also demonstrated that B-cell lymphoma 2 (Bcl-2) was the target gene of miR-34a, as demonstrated by luciferase assays. Furthermore, restoring the expression of Bcl-2 was indicated to partially block the miR-34a-induced apoptosis. Thus, data from the present study identified miR-34a as a tumor suppressor in glioma by, at least partially, targeting $\mathrm{Bcl}-2$. This may provide future novel diagnostic and therapeutic strategies for human glioma.
\end{abstract}

\section{Introduction}

Glioblastoma is a highly malignant brain tumor, characterized by poor prognosis and high recurrence rates. Despite therapeutic strategies, including surgery, radiotherapy and chemotherapy, the prognosis remains poor and the median survival of patients is only 14.6 months $(1,2)$. Thus, developments of efficient

Correspondence to: Professor Junwei Duan, Department of Neurosurgery, The Affiliated Hospital of North Sichuan Medical College, 63 Wenhua Road, Nanchong, Sichuan 637000, P.R. China

E-mail: duanjunweisc@163.com

${ }^{*}$ Contributed equally

Key words: miR-34a, apoptosis, glioma cells, Bcl-2, caspase therapeutic strategies and novel therapeutic targets are urgently required.

MicroRNAs (miRNAs) are a group of endogenous non-coding RNAs (length, $22 \mathrm{nt}$ ) that target mRNAs for cleavage or translational repression. MiRNAs induce target gene silencing via completely or partially complementing with the 3'-untranslated region (3'-UTR) of specific mRNAs and this has been demonstrated to be involved in various cellular processes (3-6). Considered to be important gene regulators, miRNAs are emerging as novel biomarkers for cancer. Previous studies have associated the dysregulation of miRNAs with the initiation and development of glioma, and a number of miRNAs are closely associated with glioma staging and patient survival, and so have been suggested to be novel diagnostic or prognostic markers (7-9). Improved understanding of miRNA-mediated effects in glioma cells may aid the future development of novel strategies in glioma diagnosis and treatment.

MicroRNA-34a (miR-34a) is a highly conserved miRNA observed in various species. In humans, there are three homologs, miR34a, miR-34b and miR-34c. MiR-34a has been demonstrated to exert a tumor suppressive effect in breast (10) and lung cancer, involving cell cycle arrest, senescence and apoptosis $(11,12)$. A previous study by Gao et al (13) has demonstrated that miR-34a was downregulated in glioma samples compared with normal brain tissue samples, and that it is also associated with glioma grade and prognosis. However, the possible roles and underlying mechanisms of miR-34a in human glioma cells remains to be elucidated. The present study aimed to demonstrate that miR-34a inhibits cell proliferation and induces cell apoptosis of U87 glioma cells by targeting B-cell lymphoma 2 (Bcl-2).

\section{Materials and methods}

Acquisition of tissue specimens. The protocol of the present study and acquisition of tissue specimens was approved by the Biomedical Research Ethics Committee of The Affiliated Hospital of North Sichuan Medical College (Nanchong, China). A total of 20 glioma tissue specimens were obtained from patients who received surgical treatment at the Department of Neurosurgery at The Affiliated Hospital of North Sichuan Medical College between June 2011 and December 2013. 
Cells. U87 cells were obtained from the Institute of Biochemistry and Cell Biology of the Chinese Academy of Sciences (Shanghai, China) and cultured in Dulbecco's modified Eagle's medium (Sigma-Aldrich, St. Louis, MO, USA) supplemented with $10 \%$ fetal bovine serum (Gibco; Thermo Fisher Scientific, Inc., Waltham, MA, USA), 100 U/ml penicillin $\mathrm{G}$ and $100 \mu \mathrm{g} / \mathrm{ml}$ streptomycin (Sigma-Aldrich). Cells were incubated in a humidified atmosphere with $5 \% \mathrm{CO}_{2}$ at $37^{\circ} \mathrm{C}$.

Cell proliferation assay. To investigate cell proliferation, U87 cells were diluted into single cell suspensions and seeded in 96-well plates $\left(1 \times 10^{4}\right.$ cells/well). Following transfection with miR-34a mimics or scramble mimics (miR-Ctrl) (Guangzhou RiboBio Co., Ltd., Guangzhou, China), $10 \mu 1$ CCK-8 was added to each well at 24, 48 and $72 \mathrm{~h}$, avoiding the production of air bubbles. The plates were incubated at $37^{\circ} \mathrm{C}$ for $1-4 \mathrm{~h}$ and the absorbance was measured at $450 \mathrm{~nm}$ using an Epoch microplate spectrophotometer (BioTek Instruments, Inc., Winooski, VT, USA). Experiments were independently repeated three times.

Cell apoptosis analysis. Apoptosis was measured using the FITC Annexin V Apoptosis Detection kit (BD Biosciences, Franklin Lakes, NJ, USA) according to the manufacturer's protocol. U87 cells transfected with miR-34a mimics or miR-Ctrl were washed and resuspended in $1 \mathrm{X}$ binding buffer at a concentration of $1 \times 10^{6}$ cells $/ \mathrm{ml}$. Cells $\left(1 \times 10^{5}\right)$ were incubated with $5 \mu \mathrm{l}$ of Annexin V-fluorescein isothiocyanate (FITC) and $5 \mu \mathrm{l}$ of propidium iodide (PI) for $15 \mathrm{~min}$ at room temperature in the dark. Following staining, cells were diluted with $400 \mu \mathrm{l}$ binding buffer and directly detected by BD FACSCalibur ${ }^{\mathrm{TM}}$ (BD Biosciences) within $1 \mathrm{~h}$. The results were analyzed using FlowJo 7.6 software (Tree Star, Inc. Ashland, OR, USA). All the cells positively stained for Annexin V-FITC were considered to be apoptotic cells and experiments were independently repeated three times.

Reverse transcription-quantitative polymerase chain reaction (RT-qPCR) analysis. For quantitative detection of miR-34a and Bcl-2, total RNA was extracted from cells or tissues using TRIzol reagent (Invitrogen; Thermo Fisher Scientific, Inc.) according to the manufacturer's protocols. RNA samples were reverse transcribed to cDNA using a PrimeScript RT Reagent Kit with gDNA Eraser (Takara Biotechnology Co., Ltd., Dalian, China) with miR-34a specific primers (5'-GTCGTATCCAGTGCAGGGTCCGAG GTATTCGCACTGGATACGACACAACCA-3'; Guangzhou RiboBio Co., Ltd.). The DNase reaction was performed by incubation with gDNA Eraser at $42^{\circ} \mathrm{C}$ for $2 \mathrm{~min}$, then RT was performed at $37^{\circ} \mathrm{C}$ for $15 \mathrm{~min}$ and $85^{\circ} \mathrm{C}$ for $10 \mathrm{~min}$. qPCR was performed using SYBR Premix Ex Taq ${ }^{\text {TM }}$ (Takara Biotechnology Co., Ltd.) in an ABI 7500 system (Applied Biosystems; Thermo Fisher Scientific, Inc.). Primers used were as follows: Sense, 5'-GCGGCGGACCGTCACAGA ATC-3' for miR-34a; sense, 5'-GCATGGGTCCCCCGA CGTTG-3' and antisense, 5'-GCTCCGGCCAGAGGC CTCAA-3' for Bcl-2; and sense, 5'-CGTGAAAAGACC CAGATCA-3' and antisense, 5'-CACAGCCTGGATGGC TACGT-3' for $\beta$-actin. The primers were obtained from
Sangon Biotech Co., Ltd. (Shanghai, China). The mRNA expression of Bcl-2 was normalized to $\beta$-actin mRNA and miR-34a was normalized to U6. The relative expression of miR-34a and Bcl-2 was quantified with the $2^{-\Delta \Delta \mathrm{Cq}}$ method (14) and experiments were independently repeated three times.

Caspase activity assays. The activities of caspase- $3,-8$ and -9 in U87 cells were measured using caspase-3, -8 and -9 colorimetric assay kits (R\&D Systems, Inc., Minneapolis, MN, USA) following the manufacturer's protocols. Briefly, U87 cells $\left(1 \times 10^{4}\right.$ cells/well) were seeded in 96-well plates and incubated for $24 \mathrm{~h}$ at $37^{\circ} \mathrm{C}$. Following transfection with miR-34a mimics or scramble mimics using Lipofectamine ${ }^{\circledR} 2000$ (Invitrogen; Thermo Fisher Scientific, Inc.), cells were washed with ice-cold phosphate-buffered saline (PBS), and the activities of caspase- $3,-8$ and -9 were determined using the kits according to the manufacturer's protocols. The colorimetric substrates DEVD-p-nitroaniline (pNA) for caspase-3, IETD-pNA for caspase- 8 or Ac-LEHD-pNA for caspase- 9 were added to the cell lysates and incubated for $1 \mathrm{~h}$ at $37^{\circ} \mathrm{C}$. The chromophore pNA was released as a result of cleavage of the substrates by caspase activity and quantified spectrophotometrically at a wavelength of $405 \mathrm{~nm}$ using the Epoch microplate spectrophotometer. Experiments were independently repeated three times.

Plasmids construction and luciferase assays. The Bcl-2 3'-UTR containing the miR-34a binding site was cloned into a modified pGL3 vector (Promega Corporation, Madison, WI, USA) immediately downstream of the luciferase gene. Mutations in the 3'-UTR of the Bcl-2 gene with miR-34a target sites deleted were generated with the QuikChange Site-Directed Mutagenesis kit (Agilent Technologies, Inc., Santa Clara, CA $<$ USA). Approximately $1 \times 10^{5}$ U87 cells/well were seeded into 24-well plates for $24 \mathrm{~h}$ prior to transfection. Cells were co-transfected with $50 \mathrm{ng}$ pGL3 firefly luciferase reporter, $10 \mathrm{ng}$ pRL-TK luciferase reporter and $50 \mathrm{nM}$ miR-34a mimics or scramble mimics using Lipofectamine ${ }^{\circledR} 2000$. Cell lysates were prepared using Passive Lysis Buffer (Promega Corporation) $48 \mathrm{~h}$ after transfection, and luciferase activity was measured using the Dual-Luciferase ${ }^{\circledR}$ Reporter assay system (Promega Corporation). Dual-Glo ${ }^{\circledR}$ Luciferase assay reagent was added to the plate $\left(75 \mu \mathrm{l} /\right.$ well). Following incubation at $20-25^{\circ} \mathrm{C}$ for $15 \mathrm{~min}$, firefly luciferase luminescence was measured by an automatic multi-mode microplate reader (Infinite $200 \mathrm{PRO}$, Tecan Group, Ltd., Männedorf, Switzerland). Subsequently, Dual-Glo ${ }^{\circledR}$ Stop\&Glo ${ }^{\circledR}$ reagent was added to the plate $(75 \mu \mathrm{l} /$ well $)$ and incubated at $20-25^{\circ} \mathrm{C}$ for $15 \mathrm{~min}$. Renilla luminescence in the same wells was detected using the Epoch microplate spectrophotometer. The ratio of firefly/Renilla luminescence was calculated for each well. The sample well ratio was normalized to the ratio from a control wells. The full length Bcl-2 gene open reading frame (ORF) was amplified using PCR. Following purification, the inserts and vector were digested by EcoRI and XhoI (New England BioLabs, Inc., Ipswich, MA, USA) at $37^{\circ} \mathrm{C}$ for $2 \mathrm{~h}$, followed by electrophoresis and extraction. Ligation and transformation was used to clone the Bcl-2 ORF into pCDNA3.1 (Invitrogen; Thermo Fisher Scientific, Inc.) to generate the pCDNA3.1-Bcl-2 construct. Successful cloning was confirmed by DNA sequencing. 
A

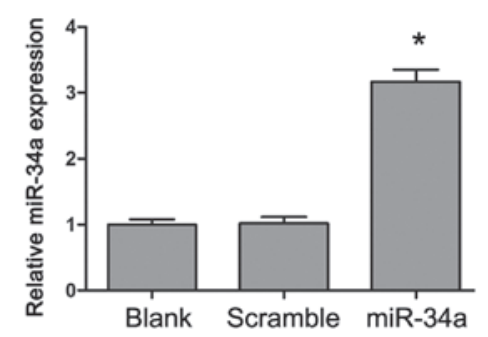

C

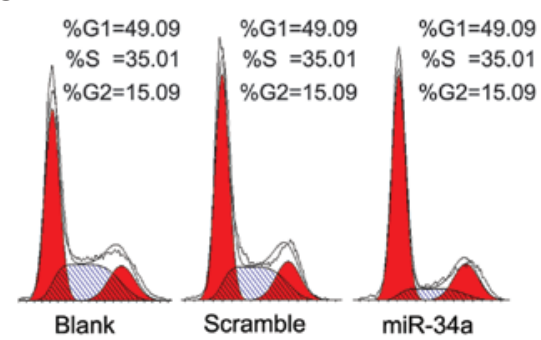

B

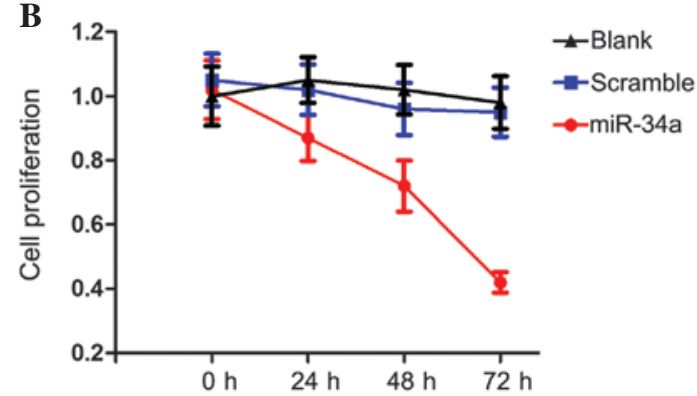

D

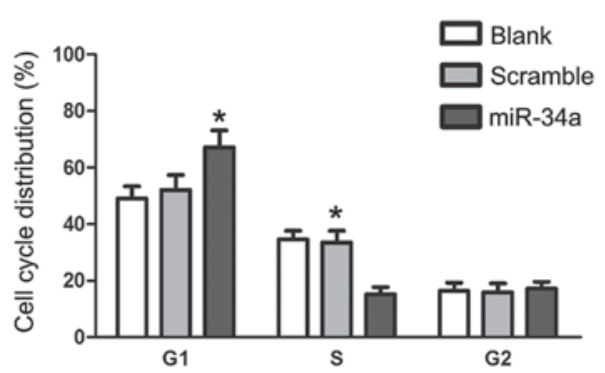

Figure 1. Effects of miR-34a on U87 cell proliferation and the cell cycle. (A) The level of miR-34a expression was detected in U87 cells upon transfection with miR-34a mimics or scramble mimics by quantitative reverse transcription-polymerase chain reaction. (B) Cell proliferation assay of U87 cells was performed following transfection with miR-34a mimics or scramble mimics using Cell Counting Kit-8. (C) Cell cycle analysis of cells following transfection was measured by propidium iodide staining and miR-34a was demonstrated to arrest the cell cycle in $\mathrm{G}_{1}$ phase. (D) Cell cycle distribution of U87 cells following transfection. The data are presented as the mean \pm standard error of the mean. ${ }^{*} \mathrm{P}<0.05$ vs. scramble control. miR-34a, microRNA-34a.

Western blot analysis. For analysis by western blotting, cells were harvested in ice-cold PBS $48 \mathrm{~h}$ after transfection. The cells were lysed on ice in cold lysis buffer (Cell Signaling Technology, Inc., Danvers, MA, USA) supplemented with protease inhibitors (Sigma-Aldrich). Protein concentrations were detected with the Bradford assay (Bio-Rad Laboratories, Inc., Hercules, CA, USA) and equal quantities of protein $(50 \mu \mathrm{g})$ were analyzed by $10 \%$ sodium dodecyl sulfate-polyacrylamide gel electrophoresis. Gels were electroblotted onto polyvinylidene difluoride membranes (EMD Millipore, Billerica, MA, USA). Following blocking with 5\% nonfat dry milk at room temperature for $2 \mathrm{~h}$, membranes were incubated with polyclonal rabbit anti-Bcl-2 (cat. no. 2872) or monoclonal rabbit anti-GAPDH (cat. no. 2118) antibodies (dilution, 1:1,000; Cell Signaling Technology, Inc.) at $4^{\circ} \mathrm{C}$ overnight. The membranes were then incubated with horseradish peroxidase-conjugated goat anti-rabbit secondary antibodies (dilution, 1:1,000; cat. no. 7074; Cell Signaling Technology, Inc.) and the protein was visualized using SuperSignal West Femto Substrate enhanced chemiluminescence kit (Pierce; Thermo Fisher Scientific, Inc.). A Bio-Rad ChemiDoc imaging system (Bio-Rad Laboratories, Inc., Hercules, CA, USA) was used to visualize the proteins. GAPDH was used as a reference gene to normalize protein expression levels. Experiments were independently repeated three times.

Statistical analysis. All data were expressed as the mean \pm the standard error of the mean from at least three independent experiments. Pearson's correlation coefficient was used to analyze the correlation between groups. Data of experiments with more than two groups were analyzed using analysis of variance and Tukey's test Results were analyzed using SPSS 16.0 software (SPSS, Inc., Chicago, IL, USA) and GraphPad
Prism 5.0 (GraphPad Software, Inc., La Jolla, CA, USA). $\mathrm{P}<0.05$ was considered to indicate a statistically significant difference.

\section{Results}

Transfection of miR-34a inhibited the proliferation of U87 cells. To investigate the association between miR-34a and glioma cell growth, miR-34a or scramble mimics were transfected into U87 cells. The miR-Ctrl is a scrambled sequence without the ability to target any human gene. The level of miR-34a was 3-4 fold higher in U87 cells following transfection with miR-34a mimics compared with the control group (Fig. 1A; P<0.05). Proliferation of U87 cells was measured using the CCK-8 assay. Overexpression of miR-34a resulted in a marked decrease of proliferation in U87 cells (Fig. 1B). The effect of miR-34a on cell cycle progression was analyzed by PI staining. The percentage of cells in the $\mathrm{S}$ phase was decreased, and the percentage of cells in $\mathrm{G}_{1}$ phase was increased $(\mathrm{P}<0.05)$ in U87 cells with miR-34a transfection (Fig. 1C and D), but not in cells transfected with the scramble mimics. The expression level of miR-34a in glioma cells inhibited cell cycle progression and proliferation.

Caspase-dependent apoptosis was induced by miR-34a in U87 cells. To investigate whether upregulation of miR-34a influenced apoptosis of U87 cells, PI/Annexin V staining was performed to detect apoptosis cells. Compared with scramble mimic-transfected cells, the proportion of Annexin-V stained cells was markedly increased $48 \mathrm{~h}$ after the transfection of miR-34a (Fig. 2A). The percentage of early apoptotic, late apoptotic and total apoptotic cells in the miR-34a-transfected U87 cells was demonstrated to be increased compared with the 
A

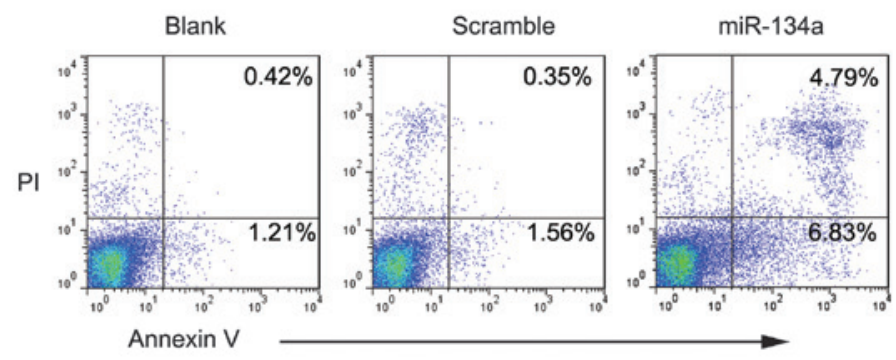

B

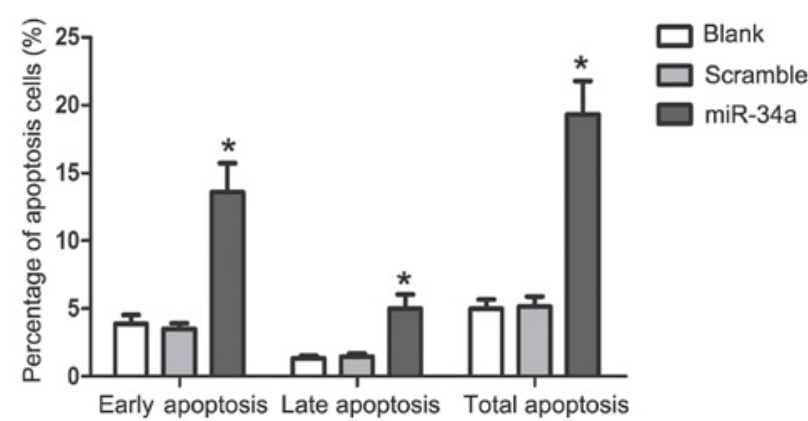

Figure 2. Effects of miR-34a on U87 cells apoptosis. (A) Analysis of cell apoptosis of U87 cells following transfection with miR-34a mimics or scramble mimics was detected by PI/Annexin-V staining. (B) The bar chart demonstrates the ratio of early apoptotic, late apoptotic and total apoptotic U87 cells $48 \mathrm{~h}$ after transfection with miR-34a or scramble mimics. The data are presented as the mean \pm standard error of the mean. $\mathrm{P}<0.05$ vs. scramble control. miR-34a, microRNA-34a; PI, propidium iodide.

A
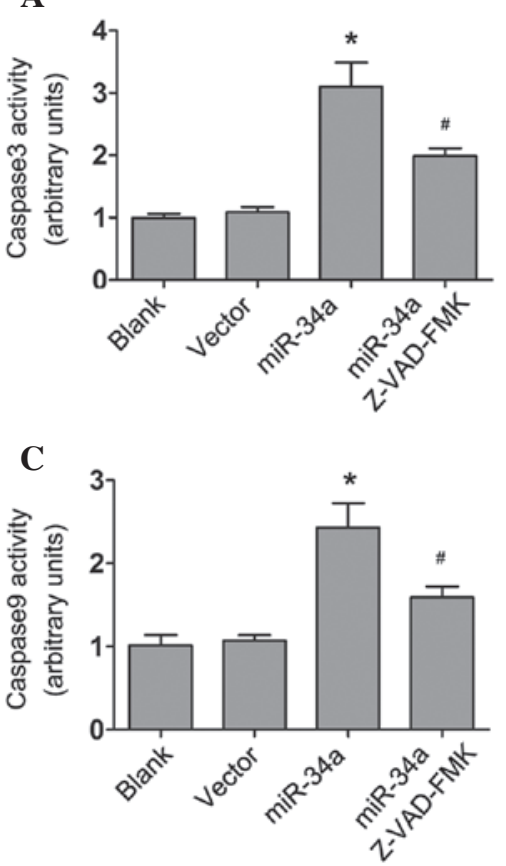

B

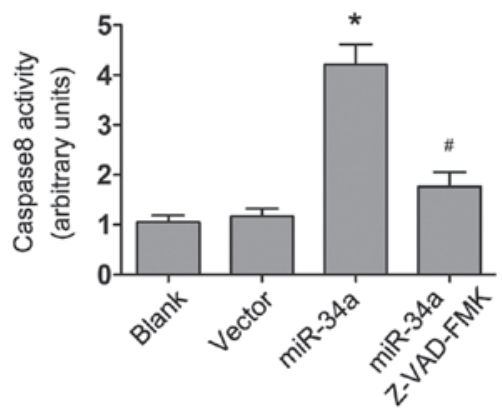

D

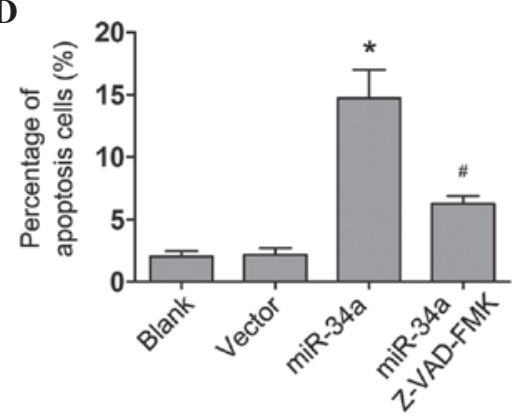

Figure 3. miR-34a induced caspase-dependent apoptosis. The activity of (A) caspase-3, (B) caspase-8 and (C) caspase-9 following transfection with miR-34a or scramble mimics and treated with Z-VAD-FMK was determined. (D) Percentage of apoptotic cells following indicated treatment. The data are presented as the mean \pm standard error of the mean $(n=3)$. ${ }^{*} \mathrm{P}<0.05$ vs. the blank group, ${ }^{\prime \prime} \mathrm{P}<0.05$ vs. the miR-34a transfected group. miR-34a, microRNA-34a.

control group (Fig. 2B; all $\mathrm{P}<0.05$ ). The activities of caspase-3, -8 and -9 significantly increased in miR-34a highly expressing cells $(\mathrm{P}<0.05)$, but not in scramble mimic-transfected cells, which suggests that miR-34a induced caspase-dependent apoptosis in U87 cells. Furthermore, U87 cells transfected with miR-34a or scramble mimics were treated with an irreversible general caspase inhibitor, z-VAD-FMK (Abcam, Cambridge, MA, US). The results demonstrated that z-VADFMK $(10 \mu \mathrm{M})$ partially reversed the effect of miR-34a, inhibiting the activity of caspase- $3,-8$ and -9 ( $\mathrm{P}<0.05$; Fig. 3 ), indicating that miR-34a induced caspase-dependent cell apoptosis.

Bcl-2 is a direct target of miR-34a in glioma cells. Bcl-2 is a critical factor in apoptosis and has an important role in the development and progression of cancer. Targetscan (www.targetscan.org) and MicroRNA (www.microrna. org) were used to search for potential miR-34a targets. The analysis demonstrated that $\mathrm{Bcl}-2$ is a theoretical target gene of miR-34a in human cells (Fig. 4A). Luciferase reporter assays were conducted to validate the prediction and Bcl-2 3'-UTR vectors and miR-34a or scramble mimics were transfected into U87 cells. As presented in Fig. 4B, a significant decrease in luciferase activity upon miR-34a transfection was observed, suggesting that miR-34a directly suppressed Bcl-2 in U87 cells $(\mathrm{P}<0.05)$. The present study also demonstrated that $\mathrm{Bcl}-2$ protein expression was specifically downregulated in miR-34a mimic-transfected cells (Fig. 4C). These data suggest that Bcl-2 is a direct target of miR-34a in glioma. Furthermore, it was observed that miR-34a levels were inversely correlated with the expression of Bcl-2 in 20 glioma tissue samples $(\mathrm{r}=-0.684, \mathrm{P}<0.001 ;$ Fig. 4D). 
A

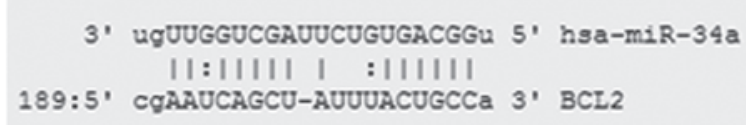

B
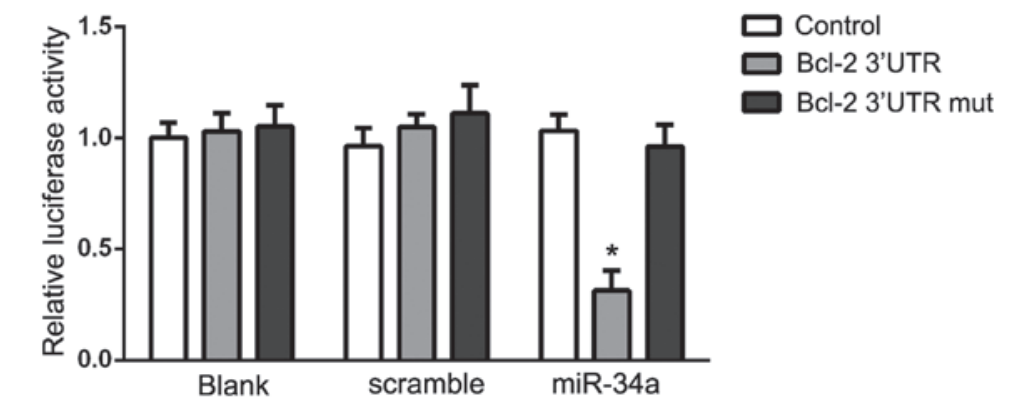

C

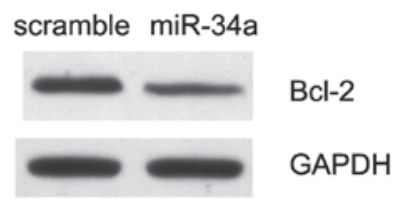

D

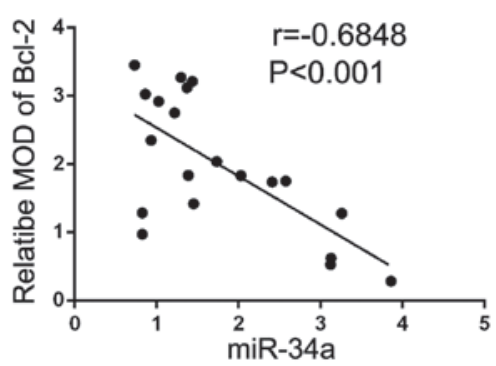

Figure 4. Bcl-2 is a functional target of miR-34a in glioma. (A) Annealing of miR-34a to Bcl-2 3'-UTR according to the microRNA.org database. (B) Bcl-2 3'-UTR or Bcl-2 3'-UTR mutation construct was co-transfected into U87 cells with miR-34a or scramble mimics and the cells were assayed for luciferase activity. (C) Bcl-2 protein expression levels were measured by western blotting in U87 cells transfected with the plasmids as indicated. (D) Correlation analysis of miR-34a and Bcl-2 protein in 20 freshly prepared human glioma tissue samples. The expression levels of miR-34a were examined using the reverse transcription-quantitative polymerase chain reaction and normalized to $5 \mathrm{~s}$ rRNA. The expression levels of Bcl-2 were quantified by immunohistochemical approach using MOD. "P<0.05 vs. scramble control. miR-34a, microRNA-34a; Bcl-2, B-cell lymphoma 2; UTR, untranslated region; MOD, mean optical density.
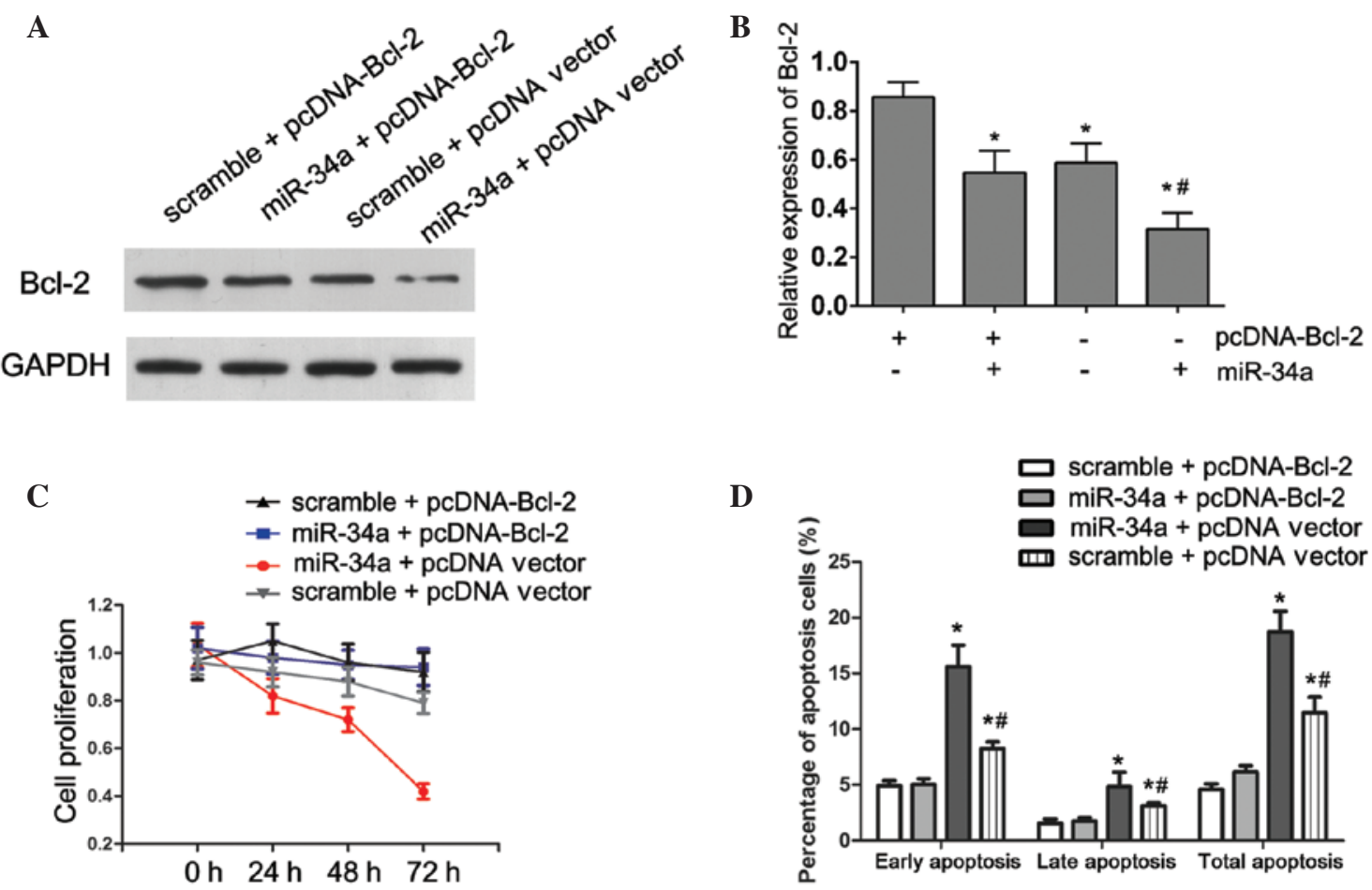

Figure 5. miR-34a induced apoptosis by targeting Bcl-2. (A) The level of Bcl-2 protein expression was detected in U87 cells co-transfected with either miR-34a mimic or scramble mimic and pcDNA-Bcl-2 or empty vector by western blotting. (B) The bar chart demonstrates the ratio of Bcl-2 protein relative to GAPDH in U87 cells, transfected with plasmids as indicated, by densitometry. (C) Cell proliferation assay of U87 cells treated as described was determined using Cell Counting Kit-8. (D) Cell apoptosis of U87 cells transfected with indicated plasmids was detected by propidium iodide/Annexin-V staining. The data are presented as the mean \pm standard error of the mean $(n=3)$. ${ }^{*} \mathrm{P}<0.05$ vs. the blank group, ${ }^{\#} \mathrm{P}<0.05$ vs. the miR-34a transfection combined with pcDNA vector group. miR-34a, microRNA-34a; Bcl-2, B-cell lymphoma 2. 
miR-34a suppressed cell proliferation and induced apoptosis partially by targeting Bcl-2. Data from the present study suggested that Bcl-2 was a target gene of miR-34a in HepG2 cells, however, the interaction between miR-34a and Bcl-2 in the regulation of cell proliferation and apoptosis in glioma cells required further elucidation. To investigate whether miR-34a mediated growth suppression in U87 cells by targeting Bcl-2, a novel construct containing the full ORF of Bcl-2 gene (pcDNA-Bcl-2). Following transfection of Bcl-2 construct into U87 cells that had been treated with miR-34a mimic for $24 \mathrm{~h}$, the expression of $\mathrm{Bcl}-2$ was rescued $(\mathrm{P}=0.014$; Fig. $5 \mathrm{~A}$ and $\mathrm{B})$. Consistent with the restored expression of $\mathrm{Bcl}-2$, increased cell proliferation (Fig. 5C), accompanied by decreased cell apoptosis (Fig. 5D) was also observed in U87 cells transfected with pcDNA-Bcl-2 constructs following the treatment with miR-34a mimics $(\mathrm{P}<0.001)$. These results demonstrated that the suppression of cell growth by miR-34a was mediated via targeting Bcl-2.

\section{Discussion}

The results from the present study support the hypothesis that miR-34a functions as a tumor suppressor in glioma cells. Bcl-2 was identified as a direct target of miR-34a in U87 cells. Restored expression of Bcl-2 in U87 cells partially reversed miR-34a-mediated apoptosis and inhibition of cell proliferation.

MiRNAs are a group of small regulatory RNAs that are involved in posttranscriptional gene expression regulation in a sequence-specific manner. MiRNAs form $\sim 1 \%$ of the genome and each of which has hundreds of different conserved or non-conserved targets, thus, they are key in various cellular processes, including invasion, proliferation and apoptosis. Increased understanding the physiological and disease-associated mechanisms of miRNAs may aid development of novel strategies for diagnosis and treatment of various diseases. Dysregulated miRNAs have been identified by microarray and associated with glioma carcinogenesis via altering expression of oncogenes or tumor suppressors to influence cell proliferation, apoptosis, and tumor cell migration and invasion. Among these miRNAs, miR-34a was reported to be underexpressed in glioma (15), however, its exact function and underlying mechanisms required further elucidation. The present study demonstrated that restored expression of miR-34a in the U87 human glioma cell line inhibited cell proliferation, arrested cell cycle progression and induced cell apoptosis. Furthermore, inhibition of caspase activity by Z-VAD-FMK suppressed the apoptosis induced by miR-34a, indicating that miR-34a inhibited the proliferation of U87 cells via the induction of caspase-dependent apoptosis.

It is well understood that the intrinsic apoptosis signaling pathway ultimately activates caspase- 3 , which may be regulated by the Bcl-2 superfamily members, including anti-apoptotic members, Bcl-2 and Bcl-xL, and pro-apoptotic members, Bax, Bak and Bid (16,17). It has been demonstrated that expression of Bcl-2 may be downregulated by miR-34a in numerous types of cells, including Min6 cells (18), PC12 cells (19) and human hepatocellular carcinoma cells (20). In the present study, the putative binding site of miR-34a in Bcl-2 3'-UTR was observed by a biological prediction program (21). Luciferase reporter assays indicated that transfection of miR-34a resulted in a marked reduction of luciferase activity by the luciferase expression constructs carrying the target Bcl-2 3'-UTR fragment, but had no effect on Bcl-2 3'-UTR mutated fragment. In addition, the protein expression of Bcl-2 was significantly decreased following treatment with miR-34a mimics demonstrating that miR-34a directly targets Bcl-2 mRNA in U87 cells. Furthermore, an inverse correlation was observed between the miR-34a level and Bcl-2 expression in human glioma tissue specimens, which was consistent with the results of Yang et al (20) in hepatocellular carcinoma. Using further rescue assays, the present study demonstrated that miR-34a induced cell cycle arrest and apoptosis was partially blocked by overexpression of $\mathrm{Bcl}-2$. These results imply that miR-34a-induced apoptosis was partially mediated by silencing of $\mathrm{Bcl}-2$.

In addition to $\mathrm{Bcl}-2$, various genes have been reported to participate in miR-34a-mediated tumor suppression, including fos-related antigen 1, platelet derived growth factor receptor and MET proto-oncogene, tyrosine receptor, were identified to be targets of miR-34a involved in tumor migration and invasion $(10,22)$. MiR-34a also modulated the sensitivity of doxorubicin-resistant MCF-7 cells to doxorubicin by directly inhibiting Notch 1 (23). Delivery of miR-34a to prostate cancer mouse models resulted in to a marked decrease in tumor growth via inhibition of the c-Myc oncogene (24). Li et al (25) demonstrated that miR-34a serves as a tumor suppressor in A172 human glioma cells, predominantly by decreasing NADPH oxidase 2 expression. The involvement of these genes in miR-34a-mediated tumor suppression in glioma remains to be investigated.

In conclusion, the results from the present study demonstrated that miR-34a was a tumor suppressor in glioma cells, suppressing cell proliferation and inducing cell apoptosis via targeting of Bcl-2. These findings aid understanding of the underlying molecular mechanisms of glioma carcinogenesis and suggest a potential use of miR-34a in future therapeutic strategies to treat glioma.

\section{Acknowledgements}

The current study was supported by Youth Innovation Project Plan of Sichuan Province Medical Scientific Research (grant no. Q15079).

\section{References}

1. Koekkoek JA, Kerkhof M, Dirven L, Heimans JJ, Reijneveld JC and Taphoorn MJ: Seizure outcome after radiotherapy and chemotherapy in low-grade glioma patients: A systematic review. Neuro Oncol 17: 924-934, 2015.

2. Chiarelli PA, Kievit FM, Zhang M and Ellenbogen RG: Bionanotechnology and the future of glioma. Surg Neurol Int 6 (Suppl 1): S45-S58, 2015.

3. Kawahara H, Imai $\mathrm{T}$ and Okano $\mathrm{H}$ : MicroRNAs in neural stem cells and neurogenesis. Front Neurosci 6: 30, 2012.

4. Di Y, Lei Y, Yu F, Changfeng F, Song W and Xuming M: MicroRNAs expression and function in cerebral ischemia reperfusion injury. J Mol Neurosci 53: 242-250, 2014.

5. Zhang Z, Chang H, Li Y, Zhang T, Zou J, Zheng X and Wu J: MicroRNAs: potential regulators involved in human anencephaly. Int J Biochem Cell Biol 42: 367-374, 2010.

6. Barger JF and Nana-Sinkam SP: MicroRNA as tools and therapeutics in lung cancer. Respir Med 109: 803-812, 2015.

7. An L, Liu Y, Wu A and Guan Y: microRNA-124 inhibits migration and invasion by down-regulating ROCK1 in glioma. PloS one 8: e69478, 2013. 
8. Li M, Li J,Liu L, Li W, Yang Y and Yuan J: MicroRNA in human glioma. Cancers 5: 1306-1331, 2013.

9. Palumbo S, Miracco C, Pirtoli L and Comincini S: Emerging roles of microRNA in modulating cell-death processes in malignant glioma. J Cell Physiol 229: 277-286, 2014.

10. Wu J, Wu G, Lv L, Ren YF, Zhang XJ, Xue YF, Li G, Lu X, Sun Z and Tang KF: MicroRNA-34a inhibits migration and invasion of colon cancer cells via targeting to Fra-1. Carcinogenesis 33: 519-528, 2012

11. Kato M, Paranjape T, Müller RU, Nallur S, Gillespie E, Keane K Esquela-Kerscher A, Weidhaas JB and Slack FJ: The mir-34 microRNA is required for the DNA damage response in vivo in C. elegans and in vitro in human breast cancer cells. Oncogene 28 2419-2424, 2009.

12. Stahlhut $\mathrm{C}$ and Slack FJ: Combinatorial action of MicroRNAs let-7 and miR-34 effectively synergizes with Erlotinib to suppress non-small cell lung cancer cell proliferation. Cell Cycle 14 2171-2180, 2015.

13. Gao H, Zhao H and Xiang W: Expression level of human miR-34a correlates with glioma grade and prognosis. J Neurooncol 113: 221-228, 2013

14. Schefe JH, Lehmann KE, Buschmann IR, Unger $T$ and Funke-Kaiser H: Quantitative real-time RT-PCR data analysis: Current concepts and the novel "gene expression's CT difference" formula. J Mol Med Berl 84: 901-910, 2006.

15. Guessous F, Zhang Y, Kofman A, Catania A, Li Y, Schiff D, Purow B and Abounader R: microRNA-34a is tumor suppressive in brain tumors and glioma stem cells. Cell Cycle 9: 1031-1036, 2010.

16. Li Y, Qi H, Li X, Hou X, Lu X and Xiao X: A novel dithiocarbamate derivative induces cell apoptosis through $\mathrm{p} 53$-dependent intrinsic pathway and suppresses the expression of the E6 oncogene of human papillomavirus 18 in HeLa cells. Apoptosis 20: 787-795, 2015.
17. Hockenbery DM, Oltvai ZN, Yin XM, Milliman CL and Korsmeyer SJ: Bcl-2 functions in an antioxidant pathway to prevent apoptosis. Cell 75: 241-251, 1993.

18. Lin X, Guan H, Huang Z, Liu J, Li H, Wei G, Cao X and Li Y Downregulation of Bcl-2 expression by miR-34a mediates palmitate-induced Min6 cells apoptosis. J Diabetes Res 2014 258695, 2014

19. Huang M, Lou D, Chang X and Zhou Z: Micro RNA alteration after paraquat induced $\mathrm{PC} 12$ cells damage and regulatory mechanism of bcl-2. Zhonghua Lao Dong Wei Sheng Zhi Ye Bing Za Zhi 32: 32-37, 2014 (In Chinese).

20. Yang F, Li QJ, Gong ZB, Zhou L, You N, Wang S, Li XL, Li JJ, An JZ, Wang DS, et al: MicroRNA-34a targets Bcl-2 and sensitizes human hepatocellular carcinoma cells to sorafenib treatment. Technol Cancer Res Treat 13: 77-86, 2014.

21. Betel D, Wilson M, Gabow A, Marks DS and Sander C: The microRNA.org resource: Targets and expression. Nucleic Acids Res 36 (Database issue): D149-D153, 2008

22. Peng Y, Guo JJ, Liu YM and Wu XL: MicroRNA-34A inhibits the growth, invasion and metastasis of gastric cancer by targeting PDGFR and MET expression. Biosci Rep 34: e00112, 2014.

23. Li XJ, Ji MH, Zhong SL, Zha QB, Xu JJ, Zhao JH and Tang JH: MicroRNA-34a modulates chemosensitivity of breast cancer cells to adriamycin by targeting Notch1. Arch Med Res 43: 514-521, 2012.

24. Yamamura S, Saini S, Majid S, Hirata H, Ueno K, Deng G and Dahiya R: MicroRNA-34a modulates c-Myc transcriptional complexes to suppress malignancy in human prostate cancer cells. PloS One 7: e29722, 2012.

25. Li SZ, Hu YY, Zhao J, Zhao YB, Sun JD, Yang YF, Ji CC, Liu ZB, Cao WD, Qu Y, et al: MicroRNA-34a induces apoptosis in the human glioma cell line, A172, through enhanced ROS production and NOX2 expression. Biochem Biophys Res Commun 444: 6-12, 2014. 